Article

\title{
Regional Enlarged Observability of Fractional Differential Equations with Riemann-Liouville Time Derivatives
}

\author{
Hayat Zouiten ${ }^{1} \mathbb{D}$, Ali Boutoulout ${ }^{1}$ and Delfim F. M. Torres ${ }^{2, * \mathbb{C}}$ \\ 1 TSI Team, MACS Laboratory, Department of Mathematics and Computer Science, Moulay Ismail University, \\ Faculty of Sciences, Meknes 11201, Morocco; zouiten.hayat1991@gmail.com (H.Z.); \\ boutouloutali@yahoo.fr (A.B.) \\ 2 Center for Research \& Development in Mathematics and Applications (CIDMA), \\ Department of Mathematics, University of Aveiro, 3810-193 Aveiro, Portugal \\ * Correspondence: delfim@ua.pt; Tel.: +351-234-370-668
}

Received: 4 November 2018; Accepted: 28 November 2018; Published: 1 December 2018

check for updates

\begin{abstract}
We introduce the concept of regional enlarged observability for fractional evolution differential equations involving Riemann-Liouville derivatives. The Hilbert Uniqueness Method (HUM) is used to reconstruct the initial state between two prescribed functions, in an interested subregion of the whole domain, without the knowledge of the state.
\end{abstract}

Keywords: fractional evolution systems; Riemann-Liouville time derivatives; enlarged observability; regional reconstruction; HUM approach

MSC: 26A33; 35R11; 93B07; 93C20

\section{Introduction}

The exact birthday of fractional calculus, and the idea of non-integer differentiation, goes back to the 17th century, precisely to 30 September 1695, when L'Hôpital wrote a question to Leibniz about the meaning of $\frac{\partial^{n}}{\partial t^{n}}$ in the case $n=\frac{1}{2}$ ([1], pp. 301-302). Since then, many great mathematicians have investigated around this question. We can mention the studies of Euler (1730), Lagrange (1772), Laplace (1812), Fourier (1822), Abel (1823), Liouville (1832), Riemann (1847), Greer (1859), Grünwald (1867), Laurent (1884), Heaviside (1892), Pincherle (1902), Marchaud (1927), Love (1938), Widder (1941), Riesz (1949), Feller (1952), among others [2]. These mathematicians began to consider how to define a fractional derivative. In 1860s, Riemann and Liouville obtained now celebrated definitions of fractional operators, by extending the Cauchy integral formula. Such fractional operators have a major role in practical problems [3]. In particular, Heymans and Podlubny have shown that it is possible to attribute a physical meaning to initial conditions expressed in terms of Riemann-Liouville fractional derivatives on the field of viscoelasticity, which is more appropriate than standard initial conditions [4]. From a mathematical point of view, fractional calculus is a generalization of the traditional differential calculus to integrals and derivatives of non-integer order.

The fact that real systems are better described with non-integer order differential equations has attracted engineers' interest, making fractional calculus a tool used nowadays in almost every area of sciences. Indeed, in the last decades, fractional calculus has been recognized as one of the best tools to describe long-memory processes and materials, anomalous diffusion, long-range interactions, long-term behaviours, power laws, and allometric scaling [5]. Such models are those described by differential equations containing fractional order derivatives. On the other hand, fractional calculus is 
especially efficient for modelling systems related to diffusion processes [6]. In $[7,8]$, the heat transfer process was successfully modelled using a fractional model based on normal and anomalous diffusion equations. In [9-11], very accurate models for ultra-capacitors and electrical energy storage elements based on diffusion and the Helmholtz effect are presented. Simultaneously, fractional calculus has played a very important role in various fields such as physics, chemistry, mechanics, electricity, economics, signal and image processing, biophysics, and bioengineering [12-14]. Likewise, for control theory, fractional calculus has an enormous role [15-17]. The reader interested in applications of fractional calculus in control and mathematical modelling of systems and processes in physics, aerodynamics, electrodynamics of complex medium, viscoelasticity, heat conduction, and electricity mechanics, is referred to $[2,18-24]$ and references therein. For numerical methods to fractional partial differential equations, see [25-27].

Despite its development, the theory of fractional differential equations, compared with the classical theory of differential equations, is a field of research only on its initial stage of development, calling great interest to many mathematicians [28-30]. For distributed parameter systems, several works deal with the problem of regional observability, which we study here in the fractional context, investigating the possibility to reconstruct the initial state or gradient only on a subregion $\omega$ of the evolution domain $\Omega$ [31-35]. For results on controllability, see [36-39]. The interest to study the concept of observability for fractional differential equations is not newee [17,40-42]. Here we investigate, for the first time in the literature, the concept of regional enlarged observability, that is, observability with constraints on the state for fractional diffusion equations. For that, we make use of the Hilbert Uniqueness Method (HUM) of Lions [43,44].

The paper is organized as follows. In Section 2, we present the problem of regional enlarged observability of fractional diffusion systems with the traditional first-order time derivative replaced by the Riemann-Liouville time fractional derivative. In Section 3, we give some preliminary results, which will be used throughout the paper. In Section 4, we characterize the enlarged observability of the system. Section 5 is focused on the regional reconstruction of the initial state in an internal subregion of the evolution domain. We present in Section 6 an example to demonstrate our main results. We end with Section 7 of conclusions and some possible directions of future research.

\section{Problem Statement}

In this section we formulate the concept of regional enlarged observability for a Riemann-Liouville time fractional diffusion system of order $\alpha \in(0,1]$. Let $\Omega$ be an open bounded subset of $\mathbb{R}^{n}(n=1,2,3)$, with a regular boundary $\partial \Omega$. For $T>0$, let us denote $Q_{T}=\Omega \times[0, T]$ and $\Sigma_{T}=\partial \Omega \times[0, T]$. We consider the following time fractional order diffusion system:

$$
\begin{cases}{ }_{0} D_{t}^{\alpha} y(x, t)=A y(x, t) & \text { in } Q_{T} \\ y(\xi, t)=0 & \text { on } \Sigma_{T} \\ \lim _{t \rightarrow 0^{+}}{ }_{0} I_{t}^{1-\alpha} y(x, t)=y_{0}(x) & \text { in } \Omega\end{cases}
$$

where ${ }_{0} D_{t}^{\alpha}$ and ${ }_{0} I_{t}^{\alpha}$ denote, respectively, the (left) Riemann-Liouville fractional derivative and integral with respect to time $t$, with $\alpha \in \mathbb{R}$ such that $0<\alpha \leq 1$. For details on these operators, see, e.g., $[20,24,25,45]$. Here we just recall their definition:

$$
{ }_{0} D_{t}^{\alpha} y(x, t)=\frac{\partial}{\partial t}{ }_{0} I_{t}^{1-\alpha} y(x, t)
$$

and

$$
{ }_{0} I_{t}^{\alpha} y(x, t)=\frac{1}{\Gamma(\alpha)} \int_{0}^{t}(t-s)^{\alpha-1} y(x, s) d s,
$$

where $\Gamma(\alpha)$ denotes Euler's Gamma function. The second order operator $A$ in (1) is linear with dense domain, such that the coefficients do not depend on time $t$ and generates a strongly continuous 
semi-group $(S(t))_{t \geq 0}$ on the Hilbert space $L^{2}(\Omega)$. We assume that the initial state $y_{0} \in L^{2}(\Omega)$ is unknown. The observation space is $\mathcal{O}=L^{2}\left(0, T ; \mathbb{R}^{q}\right)$.

Without loss of generality, we denote $y(\cdot, t):=y(t)$. The measurements are obtained by the output function given by

$$
z(t)=C y(t), \quad t \in[0, T],
$$

where $C$ is called the observation operator, which is a linear operator (possibly unbounded), depending on the structure and the number $q \in \mathbb{N}$ of the considered sensors, with dense domain $D(C) \subseteq L^{2}(\Omega)$ and range in $\mathcal{O}$.

\section{Preliminaries}

In this section, we recall some results for Riemann-Liouville time fractional differential systems and some notions and results to be used thereafter.

Lemma 1 (See $[36,46,47])$. For any $u_{0} \in L^{2}(\Omega), 0<\alpha \leq 1$, we say that the function $u \in L^{2}\left(0, T ; L^{2}(\Omega)\right)$ is a mild solution of the system

$$
\left\{\begin{array}{l}
{ }_{0} D_{t}^{\alpha} u(t)=A u(t), \quad t \in[0, T], \\
\lim _{t \rightarrow 0^{+}}{ }_{0} I_{t}^{1-\alpha} u(t)=u_{0}
\end{array}\right.
$$

if $u$ satisfies the equation

$$
u(t)=H_{\alpha}(t) u_{0}
$$

where

$$
\begin{gathered}
H_{\alpha}(t)=\alpha t^{\alpha-1} \int_{0}^{\infty} \theta \xi_{\alpha}(\theta) S\left(t^{\alpha} \theta\right) d \theta, \\
\xi_{\alpha}(\theta)=\frac{1}{\alpha} \theta^{-1-\frac{1}{\alpha}} \omega_{\alpha}\left(\theta^{-\frac{1}{\alpha}}\right), \\
\omega_{\alpha}(\theta)=\frac{1}{\pi} \sum_{n=1}^{\infty}(-1)^{n-1} \theta^{-n \alpha-1} \frac{\Gamma(n \alpha+1)}{n !} \sin (n \pi \alpha), \quad \theta \in(0, \infty),
\end{gathered}
$$

with $\xi_{\alpha}$ the probability density function defined on $(0, \infty)$, satisfying

$$
\xi_{\alpha}(\theta) \geq 0, \quad \theta \in(0, \infty) \text { and } \int_{0}^{\infty} \xi_{\alpha}(\theta) d \theta=1 .
$$

Moreover,

$$
\int_{0}^{\infty} \theta^{v} \xi_{\alpha}(\theta) d \theta=\frac{\Gamma(1+v)}{\Gamma(1+\alpha v)}, \quad v \geq 0
$$

Note that a mild solution of system (1) can be written as

$$
y(x, t)=H_{\alpha}(t) y_{0}, \quad t \in[0, T] .
$$

In order to prove our results, the following lemma is used.

Lemma 2 (See [48]). Let the reflection operator $\mathcal{Q}$ on the interval $[0, T]$ be defined by

$$
\mathcal{Q} f(t):=f(T-t),
$$

for some function $f$ that is differentiable and integrable in the Riemann-Liouville sense. Then the following relations hold:

$$
\mathcal{Q}_{0} D_{t}^{\alpha} f(t)={ }_{t} D_{T}^{\alpha} \mathcal{Q} f(t), \quad \mathcal{Q}_{0} I_{t}^{\alpha} f(t)={ }_{t} I_{T}^{\alpha} \mathcal{Q} f(t)
$$


and

$$
{ }_{0} D_{t}^{\alpha} \mathcal{Q} f(t)=\mathcal{Q}_{t} D_{T}^{\alpha} f(t), \quad{ }_{0} I_{t}^{\alpha} \mathcal{Q} f(t)=\mathcal{Q}_{t} I_{T}^{\alpha} f(t) .
$$

Follows some notions of admissibility of the output operator $C$. The output function of the autonomous system (1) is expressed by

$$
z(t)=C H_{\alpha}(t) y_{0}=K_{\alpha}(t) y_{0}, \quad t \in[0, T]
$$

where $K_{\alpha}: L^{2}(\Omega) \longrightarrow \mathcal{O}$ is a linear operator. To obtain the adjoint operator of $K_{\alpha}$, we have two cases.

Case 1. $C$ is bounded (e.g., zone sensors). Let $C: L^{2}(\Omega) \longrightarrow \mathcal{O}$ and $C^{*}$ be its adjoint. We get that the adjoint operator of $K_{\alpha}$ can be given by

$$
\begin{aligned}
K_{\alpha}^{*}: \mathcal{O} & \longrightarrow L^{2}(\Omega) \\
z^{*} & \longrightarrow \int_{0}^{T} H_{\alpha}^{*}(s) C^{*} z^{*}(s) d s .
\end{aligned}
$$

Case 2. $C$ is unbounded (e.g., pointwise sensors). In this case, we have

$$
C: D(C) \subseteq L^{2}(\Omega) \longrightarrow \mathcal{O}
$$

with $C^{*}$ denoting its adjoint. In order to give a sense to (2), we make the assumption that $C$ is an admissible observation operator in the sense of Definition 1.

Definition 1. The operator $C$ of system (1) and (2) is an admissible observation operator if there exists a constant $M>0$ such that

$$
\int_{0}^{T}\left\|C H_{\alpha}(s) y_{0}\right\|^{2} d s \leq M\left\|y_{0}\right\|^{2}
$$

for any $y_{0} \in D(C)$.

Note that the admissibility of $C$ guarantees that we can extend the mapping

$$
y_{0} \longmapsto C H_{\alpha}(t) y_{0}=K_{\alpha}(t) y_{0}
$$

to a bounded linear operator from $L^{2}(\Omega)$ to $\mathcal{O}$. For more details, see, e.g., [49-51]. Then the adjoint of the operator $K_{\alpha}$ can be defined as

$$
\begin{aligned}
K_{\alpha}^{*}: D\left(K_{\alpha}^{*}\right) \subseteq \mathcal{O} & \longrightarrow L^{2}(\Omega) \\
z^{*} & \longrightarrow \int_{0}^{T} H_{\alpha}^{*}(s) C^{*} z^{*}(s) d s .
\end{aligned}
$$

\section{Enlarged Observability and Characterization}

Let $\omega$ be a subregion of $\Omega$ with a positive Lebesgue measure. We define the restriction operator $\chi_{\omega}$ and its adjoint $\chi_{\omega}^{*}$ by

$$
\begin{aligned}
\chi_{\omega}: L^{2}(\Omega) & \longrightarrow L^{2}(\omega) \\
y & \longrightarrow \chi_{\omega} y=y_{\mid \omega}
\end{aligned}
$$

and

$$
\left(\chi_{\omega}^{*} y\right)(x)= \begin{cases}y(x) & \text { if } x \in \omega, \\ 0 & \text { if } x \in \Omega \backslash \omega .\end{cases}
$$

Similarly to the discussions in $[32,49,52]$, it follows that a necessary and sufficient condition for the regional exact observability of the system described by (1) and (2) in $\omega$ at time $t$ is that $\operatorname{Im}\left(\chi_{\omega} K_{\alpha}^{*}\right)=L^{2}(\omega)$. 
Let $\beta(\cdot)$ and $\gamma(\cdot)$ be two functions defined in $L^{2}(\omega)$ such that $\beta(\cdot) \leq \gamma(\cdot)$ almost everywhere (a.e.) in $\omega$. Throughout the paper, we set

$$
[\beta(\cdot), \gamma(\cdot)]=\left\{y \in L^{2}(\omega) \mid \beta(\cdot) \leq y(\cdot) \leq \gamma(\cdot) \text { a.e. in } \omega\right\} .
$$

We consider

$$
y_{0}= \begin{cases}y_{0}^{1} & \text { in }[\beta(\cdot), \gamma(\cdot)], \\ y_{0}^{2} & \text { in } L^{2}(\Omega) \backslash[\beta(\cdot), \gamma(\cdot)] .\end{cases}
$$

The study of regional enlarged observability for the Riemann-Liouville time fractional order diffusion system amounts to solving the following problem.

Problem 1. Given the system (1) together with the output (2) in $\omega$ at time $t \in[0, T]$, is it possible to reconstruct $y_{0}^{1}$ between two prescribed functions $\beta(\cdot)$ and $\gamma(\cdot)$ in $\omega$ ?

Before proving our first result, we need two important definitions.

Definition 2. The system (1) together with the output (2) is said to be exactly $[\beta(\cdot), \gamma(\cdot)]$-observable in $\omega$ if

$$
\operatorname{Im}\left(\chi_{\omega} K_{\alpha}^{*}\right) \cap[\beta(\cdot), \gamma(\cdot)] \neq \varnothing .
$$

Definition 3. The sensor $(D, f)$ is said to be exactly $[\beta(\cdot), \gamma(\cdot)]$-strategic in $\omega$ if the observed system is exactly $[\beta(\cdot), \gamma(\cdot)]$-observable in $\omega$.

Remark 1. If $\alpha=1$, then system (1) is reduced to the normal diffusion process recently considered in [53]. The results of [53] are a particular case of our results.

Remark 2. If the system (1) together with the output (2) is exactly $[\beta(\cdot), \gamma(\cdot)]$-observable in $\omega_{1}$, then it is exactly $[\beta(\cdot), \gamma(\cdot)]$-observable in any subregion $\omega_{2} \subset \omega_{1}$.

Theorem 1. The following two statements are equivalent:

1. The system (1) together with the output (2) is exactly $[\beta(\cdot), \gamma(\cdot)]$-observable in $\omega$.

2. $\operatorname{Ker}\left(K_{\alpha} \chi_{\omega}^{*}\right) \cap[\beta(\cdot), \gamma(\cdot)]=\{0\}$.

Proof. We begin by proving that statement 1 implies 2 . For that we show that

$$
\operatorname{Im}\left(\chi_{\omega} K_{\alpha}^{*}\right) \cap[\beta(\cdot), \gamma(\cdot)] \neq \varnothing \Longrightarrow \operatorname{Ker}\left(K_{\alpha} \chi_{\omega}^{*}\right) \cap[\beta(\cdot), \gamma(\cdot)]=\{0\} .
$$

Suppose that

$$
\operatorname{Ker}\left(K_{\alpha} \chi_{\omega}^{*}\right) \cap[\beta(\cdot), \gamma(\cdot)] \neq\{0\} .
$$

Let us consider $y \in \operatorname{Ker}\left(K_{\alpha} \chi_{\omega}^{*}\right) \cap[\beta(\cdot), \gamma(\cdot)]$ such that $y \neq 0$. Then, $y \in \operatorname{Ker}\left(K_{\alpha} \chi_{\omega}^{*}\right)$ and $y \in$ $[\beta(\cdot), \gamma(\cdot)]$. We have $\operatorname{Ker}\left(K_{\alpha} \chi_{\omega}^{*}\right)=\operatorname{Im}\left(\chi_{\omega} K_{\alpha}^{*}\right)^{\perp}$, so that $y \in \operatorname{Im}\left(\chi_{\omega} K_{\alpha}^{*}\right)^{\perp}, y \neq 0$. Therefore, $y \notin$ $\operatorname{Im}\left(\chi_{\omega} K_{\alpha}^{*}\right)$, and

$$
\begin{gathered}
\operatorname{Ker}\left(K_{\alpha} \chi_{\omega}^{*}\right) \cap[\beta(\cdot), \gamma(\cdot)] \subset L^{2}(\omega) \backslash \operatorname{Im}\left(\chi_{\omega} K_{\alpha}^{*}\right), \\
\operatorname{Im}\left(\chi_{\omega} K_{\alpha}^{*}\right) \subset\left[L^{2}(\omega) \backslash \operatorname{Ker}\left(K_{\alpha} \chi_{\omega}^{*}\right)\right] \cup\left[L^{2}(\omega) \backslash[\beta(\cdot), \gamma(\cdot)]\right] .
\end{gathered}
$$

We have

$$
\operatorname{Im}\left(\chi_{\omega} K_{\alpha}^{*}\right) \subset L^{2}(\omega) \backslash \operatorname{Ker}\left(K_{\alpha} \chi_{\omega}^{*}\right) .
$$

Accordingly,

$$
\operatorname{Im}\left(\chi_{\omega} K_{\alpha}^{*}\right) \cap \operatorname{Ker}\left(K_{\alpha} \chi_{\omega}^{*}\right)=\varnothing
$$


and

$$
\operatorname{Im}\left(\chi_{\omega} K_{\alpha}^{*}\right) \cap \operatorname{Im}\left(\chi_{\omega} K_{\alpha}^{*}\right)^{\perp}=\varnothing,
$$

which is absurd. Since

$$
\operatorname{Im}\left(\chi_{\omega} K_{\alpha}^{*}\right) \subset L^{2}(\omega) \backslash[\beta(\cdot), \gamma(\cdot)],
$$

it follows that

$$
\operatorname{Im}\left(\chi_{\omega} K_{\alpha}^{*}\right) \cap[\beta(\cdot), \gamma(\cdot)]=\varnothing,
$$

which is also absurd. Consequently,

$$
\operatorname{Ker}\left(K_{\alpha} \chi_{\omega}^{*}\right) \cap[\beta(\cdot), \gamma(\cdot)]=\{0\} .
$$

We now prove the reverse implicationtatement 2 implies 1 . For that we show that

$$
\operatorname{Ker}\left(K_{\alpha} \chi_{\omega}^{*}\right) \cap[\beta(\cdot), \gamma(\cdot)]=\{0\} \quad \Longrightarrow \quad \operatorname{Im}\left(\chi_{\omega} K_{\alpha}^{*}\right) \cap[\beta(\cdot), \gamma(\cdot)] \neq \varnothing .
$$

Suppose that

$$
\operatorname{Ker}\left(K_{\alpha} \chi_{\omega}^{*}\right) \cap[\beta(\cdot), \gamma(\cdot)]=\{0\}
$$

Let us consider

$$
y \in \operatorname{Ker}\left(K_{\alpha} \chi_{\omega}^{*}\right) \cap[\beta(\cdot), \gamma(\cdot)] .
$$

Then, $y \in \operatorname{Ker}\left(K_{\alpha} \chi_{\omega}^{*}\right)$ and $y \in[\beta(\cdot), \gamma(\cdot)]$ such that $y=0$. We have

$$
\operatorname{Ker}\left(K_{\alpha} \chi_{\omega}^{*}\right)=\operatorname{Im}\left(\chi_{\omega} K_{\alpha}^{*}\right)^{\perp},
$$

so $y \in \operatorname{Im}\left(\chi_{\omega} K_{\alpha}^{*}\right)^{\perp}$ such that $y=0$. Hence,

$$
y \in \operatorname{Im}\left(\chi_{\omega} K_{\alpha}^{*}\right) \text { and } y \in[\beta(\cdot), \gamma(\cdot)]
$$

and

$$
\operatorname{Im}\left(\chi_{\omega} K_{\alpha}^{*}\right) \cap[\beta(\cdot), \gamma(\cdot)] \neq \varnothing,
$$

which shows that (1) and (2) is exactly $[\beta(\cdot), \gamma(\cdot)]$-observable in $\omega$.

\section{The HUM Approach}

The purpose of this section is to present an approach that allows us to reconstruct the initial state of the system (1) between two prescribed functions $\beta(\cdot)$ and $\gamma(\cdot)$ in $\omega$. Our approach constitutes an extension of the Hilbert Uniqueness Method (HUM) developed by Lions [43,44]. In what follows, $\mathcal{G}$ is defined by

$$
\mathcal{G}=\left\{g \in L^{2}(\Omega) \mid g=0 \text { in } L^{2}(\Omega) \backslash[\beta(\cdot), \gamma(\cdot)]\right\} .
$$

\subsection{Pointwise Sensors}

Let us consider system (1) observed by a pointwise sensor $\left(b, \delta_{b}\right)$, where $b \in \bar{\Omega}$ is the sensor location and $\delta$ is the Dirac mass concentrated in $b$. For details on pointwise sensors we refer the reader to [33]. Here the output function is given by

$$
z(t)=\varphi(b, T-t), \quad t \in[0, T] .
$$


For $\varphi_{0} \in \mathcal{G}$, we consider the following system:

$$
\begin{cases}{ }_{0} D_{t}^{\alpha} \varphi(x, t)=A \varphi(x, t) & \text { in } Q_{T} \\ \varphi(\xi, t)=0 & \text { on } \Sigma_{T} \\ \lim _{t \rightarrow 0^{+}}{ }_{0} I_{t}^{1-\alpha} \varphi(x, t)=\varphi_{0}(x) & \text { in } \Omega .\end{cases}
$$

Without loss of generality, we denote $\varphi(x, t):=\varphi(t)$. System (5) admits a unique solution $\varphi \in L^{2}\left(0, T ; H_{0}^{1}(\Omega)\right) \cap C(\Omega \times[0, T])$ given by $\varphi(t)=H_{\alpha}(t) \varphi_{0}$. We consider a semi-norm on $\mathcal{G}$ defined by

$$
\varphi_{0} \longmapsto\left\|\varphi_{0}\right\|_{\mathcal{G}}^{2}=\int_{0}^{T}\|C \varphi(T-t)\|^{2} d t .
$$

The following result holds.

Lemma 3. If the system (1) together with the output (4) is exactly $[\beta(\cdot), \gamma(\cdot)]$-observable in $\omega$, then (6) defines a norm on $\mathcal{G}$.

Proof. Consider $\varphi_{0} \in \mathcal{G}$. Then,

$$
\left\|\varphi_{0}\right\|_{\mathcal{G}} \quad \Longrightarrow \quad C \varphi(T-t)=0 \text { for all } t \in[0, T] .
$$

We have

$$
\varphi_{0} \in L^{2}(\Omega) \quad \Longrightarrow \quad \chi_{\omega} \varphi_{0} \in L^{2}(\omega)
$$

or

$$
K_{\alpha}(t) \chi_{\omega}^{*} \chi_{\omega} \varphi_{0}=C H_{\alpha}(t) \chi_{\omega}^{*} \chi_{\omega} \varphi_{0}=0 .
$$

Hence,

$$
\chi_{\omega} \varphi_{0} \in \operatorname{Ker}\left(K_{\alpha} \chi_{\omega}^{*}\right) .
$$

For $\chi_{\omega} \varphi_{0} \in[\beta(\cdot), \gamma(\cdot)]$, one has $\chi_{\omega} \varphi_{0} \in \operatorname{Ker}\left(K_{\alpha} \chi_{\omega}^{*}\right) \cap[\beta(\cdot), \gamma(\cdot)]$ and, because the system is exactly $[\beta(\cdot), \gamma(\cdot)]$-observable in $\omega, \chi_{\omega} \varphi_{0}=0$. Consequently, $\varphi_{0}=0$ and (6) is a norm.

Consider the system

$$
\begin{cases}\mathcal{Q}{ }_{t} D_{T}^{\alpha} \Psi(x, t)=A^{*} \mathcal{Q} \Psi(x, t)+C^{*} C \mathcal{Q} \varphi(x, t) & \text { in } Q_{T}, \\ \Psi(\xi, t)=0 & \text { on } \Sigma_{T}, \\ \lim _{t \rightarrow T^{-}} \mathcal{Q}_{t} I_{T}^{1-\alpha} \Psi(x, t)=0 & \text { in } \Omega,\end{cases}
$$

controlled by the solution of (5). For $\varphi_{0} \in \mathcal{G}$, we define the operator $\Lambda: \mathcal{G} \longrightarrow \mathcal{G}^{*}$ by

$$
\Lambda \varphi_{0}=\mathcal{P}(\Psi(0))
$$

where $\mathcal{P}=\chi_{\omega}^{*} \chi_{\omega}$ and $\Psi(0)=\Psi(x, 0)$. Let us now consider the system

$$
\begin{cases}\mathcal{Q}{ }_{t} D_{T}^{\alpha} \Theta(x, t)=A^{*} \mathcal{Q} \Theta(x, t)+C^{*} \mathcal{Q} z(t) & \text { in } Q_{T}, \\ \Theta(\xi, t)=0 & \text { on } \Sigma_{T}, \\ \lim _{t \rightarrow T^{-}} \mathcal{Q}_{t} I_{T}^{1-\alpha} \Theta(x, t)=0 & \text { in } \Omega .\end{cases}
$$

If $\varphi_{0}$ is chosen such that $\Theta(0)=\Psi(0)$ in $\omega$, then system (8) can be seen as the adjoint of system (1) and our problem of enlarged observability is reduced to solve the equation

$$
\Lambda \varphi_{0}=\mathcal{P}(\Theta(0)) .
$$


Theorem 2. If system (1) together with the output (4) is exactly $[\beta(\cdot), \gamma(\cdot)]$-observable in $\omega$, then equation (9) admits a unique solution $\varphi_{0} \in \mathcal{G}$, which coincides with the initial state $y_{0}^{1}$ observed between $\beta(\cdot)$ and $\gamma(\cdot)$ in $\omega$. Moreover, $y_{0}^{1}=\chi_{\omega} \varphi_{0}$.

Proof. By Lemma 1 , we see that $\|\cdot\|_{\mathcal{G}}$ is a norm of the space $\mathcal{G}$ provided that the system (1) together with the output (4) is exactly $[\beta(\cdot), \gamma(\cdot)]$-observable in $\omega$. Now, we show that (9) admits a unique solution in $\mathcal{G}$. For any $\varphi_{0} \in \mathcal{G}$, Equation (9) admits a unique solution if $\Lambda$ is an isomorphism. Then,

$$
\begin{aligned}
\left\langle\Lambda \varphi_{0}, \varphi_{0}\right\rangle_{L^{2}(\Omega)} & =\left\langle\mathcal{P} \Psi(0), \varphi_{0}\right\rangle_{L^{2}(\Omega)} \\
& =\left\langle\chi_{\omega}^{*} \chi_{\omega} \Psi(0), \varphi_{0}\right\rangle_{L^{2}(\Omega)} \\
& =\left\langle\Psi(0), \varphi_{0}\right\rangle_{L^{2}(\omega)}
\end{aligned}
$$

or $\Psi(t)$ is the solution of system (7), that is,

$$
\Psi(t)=H_{\alpha}^{*}(T-t) \Psi(T)+\int_{t}^{T} H_{\alpha}^{*}(T-\tau) C^{*} C \varphi(T-\tau) d \tau
$$

and

$$
\Psi(0)=\int_{0}^{T} H_{\alpha}^{*}(T-\tau) C^{*} C \varphi(T-\tau) d \tau,
$$

where

$$
H_{\alpha}^{*}(t)=\alpha t^{\alpha-1} \int_{0}^{\infty} \theta \xi_{\alpha}(\theta) S^{*}\left(t^{\alpha} \theta\right) d \theta
$$

with $\left(S^{*}(t)\right)_{t \geq 0}$ the strongly continuous semi-group generated by $A^{*}$. We obtain that

$$
\begin{aligned}
\left\langle\Lambda \varphi_{0}, \varphi_{0}\right\rangle_{L^{2}(\Omega)} & =\left\langle\Psi(0), \varphi_{0}\right\rangle \\
& =\left\langle\int_{0}^{T} H_{\alpha}^{*}(T-\tau) C^{*} C \varphi(T-\tau) d \tau, \varphi_{0}\right\rangle \\
& =\int_{0}^{T}\left\langle C \varphi(T-\tau), C H_{\alpha}(T-\tau) \varphi_{0}\right\rangle d \tau \\
& =\int_{0}^{T}\|C \varphi(T-\tau)\|^{2} d \tau \\
& =\left\|\varphi_{0}\right\|_{\mathcal{G}}^{2}
\end{aligned}
$$

concluding that $\Lambda$ is an isomorphism. Consequently, Equation (9) has a unique solution that is also the initial state to be estimated between $\beta(\cdot)$ and $\gamma(\cdot)$ in the subregion $\omega$ given by

$$
y_{0}^{1}=\chi_{\omega} \varphi_{0} .
$$

The proof is complete.

\subsection{Zone Sensors}

Let us come back to system (1) and suppose that the measurements are given by an internal zone sensor defined by $(D, f)$ with $D \subset \Omega$ and $f \in L^{2}(D)$. The system is augmented with the output function

$$
z(t)=\int_{D} y(x, T-t) f(x) d x
$$

In this case, we consider (5), $\mathcal{G}$ given by (3), and we define a semi-norm on $\mathcal{G}$ by

$$
\left\|\varphi_{0}\right\|_{\mathcal{G}}^{2}=\int_{0}^{T}\langle\varphi(T-t), f\rangle_{L^{2}(D)}^{2} d t
$$


with

$$
\begin{cases}\mathcal{Q}{ }_{t} D_{T}^{\alpha} \Psi(x, t)=A^{*} \mathcal{Q} \Psi(x, t)+\langle\mathcal{Q} \varphi(t), f\rangle_{L^{2}(D)} \chi_{D} f(x) & \text { in } Q_{T} \\ \Psi(\xi, t)=0 & \text { on } \Sigma_{T} \\ \lim _{t \rightarrow T^{-}} \mathcal{Q}{ }_{t} I_{T}^{1-\alpha} \Psi(x, t)=0 & \text { in } \Omega .\end{cases}
$$

We introduce the operator

$$
\begin{aligned}
\Lambda: \mathcal{G} & \longrightarrow \mathcal{G}^{*} \\
\varphi_{0} & \longrightarrow \Lambda \varphi_{0}=\mathcal{P}(\Psi(0)),
\end{aligned}
$$

where $\mathcal{P}=\chi_{\omega}^{*} \chi_{\omega}$ and $\Psi(0)=\Psi(x, 0)$. Let us consider the system

$$
\begin{cases}\mathcal{Q}{ }_{t} D_{T}^{\alpha} \Theta(x, t)=A^{*} \mathcal{Q} \Theta(x, t)+\langle\mathcal{Q} z(t), f\rangle_{L^{2}(D)} \chi_{D} f(x) & \text { in } Q_{T}, \\ \Theta(\xi, t)=0 & \text { on } \Sigma_{T}, \\ \lim _{t \rightarrow T^{-}} \mathcal{Q}{ }_{t} I_{T}^{1-\alpha} \Theta(x, t)=0 & \text { in } \Omega .\end{cases}
$$

If $\varphi_{0}$ is chosen such that $\Theta(0)=\Psi(0)$ in $\omega$, then (13) can be seen as the adjoint of system (1) and our problem of enlarged observability consists to solve the equation

$$
\Lambda \varphi_{0}=\mathcal{P}(\Theta(0))
$$

Theorem 3. If system (1) together with the output (10) is exactly $[\beta(\cdot), \gamma(\cdot)]$-observable in $\omega$, then Equation (14) has a unique solution $\varphi_{0} \in \mathcal{G}$, which coincides with the initial state $y_{0}^{1}$ observed between $\beta(\cdot)$ and $\gamma(\cdot)$ in $\omega$.

Proof. The proof is similar to the proof of Theorem 2.

\section{Example}

Let us consider the following one-dimensional time fractional differential system of order $\alpha \in(0,1]$ in $\Omega_{1}=[0,1]$, excited by a pointwise sensor:

$$
\left\{\begin{array}{lll}
{ }_{0} D_{t}^{\alpha} y(x, t)=\frac{\partial^{2} y(x, t)}{\partial x^{2}} & \text { in } & {[0,1] \times[0, T]} \\
y(0, t)=y(1, t)=0 & \text { in } & {[0, T]} \\
\lim _{t \rightarrow 0^{+}}{ }_{0} I_{t}^{1-\alpha} y(x, t)=y_{0}(x) & \text { in } & {[0,1]}
\end{array}\right.
$$

augmented with the output function

$$
z(t)=C y(x, t)=y(b, t)
$$

where $b=\frac{1}{2} \in \Omega_{1}$. The operator $A=\frac{\partial^{2}}{\partial x^{2}}$ has a complete set of eigenfunctions $\left(\varphi_{k}\right)$ in $L^{2}\left(\Omega_{1}\right)$ associated with the eigenvalues $\left(\lambda_{k}\right)$, given by

$$
\varphi_{k}(x)=\sqrt{2} \sin (k \pi x) \text { and } \lambda_{k}=-k^{2} \pi^{2}
$$

with

$$
S(t) y(x)=\sum_{k=1}^{\infty} e^{\lambda_{k} t}\left\langle y, \varphi_{k}\right\rangle_{L^{2}\left(\Omega_{1}\right)} \varphi_{k}(x) .
$$


Then,

$$
H_{\alpha}(t) y(x)=\sum_{k=1}^{\infty} t^{\alpha-1} E_{\alpha, \alpha}\left(\lambda_{k} t^{\alpha}\right)\left\langle y, \varphi_{k}\right\rangle_{L^{2}\left(\Omega_{1}\right)} \varphi_{k}(x),
$$

where $E_{\alpha, \alpha^{\prime}}(z):=\sum_{k=0}^{\infty} \frac{z^{k}}{\Gamma\left(\alpha k+\alpha^{\prime}\right)}$, $\operatorname{Re} \alpha>0, \alpha^{\prime}, z \in \mathbb{C}$, is the generalized Mittag-Leffler function in two parameters (see, e.g., [54]). Let $y_{0}(x)=\sin (2 \pi x)$ be the initial state to be observed. Then, for $\omega_{1}=\left[\frac{1}{6}, \frac{1}{3}\right]$, the following result holds.

Proposition 1. There is a state for which the system (15) and (16) is not weakly observable in $\Omega_{1}$ but it is exactly $\left[\beta_{1}(\cdot), \gamma_{1}(\cdot)\right]$-observable in $\omega_{1}$.

Proof. To show that system (15) and (16) is not weakly observable in $\Omega_{1}$, it is sufficient to verify that $y_{0} \in \operatorname{Ker}\left(K_{\alpha}\right)$. We have

$$
\begin{aligned}
K_{\alpha} y_{0}(x) & =\sum_{i=1}^{\infty} t^{\alpha-1} E_{\alpha, \alpha}\left(\lambda_{i} t^{\alpha}\right)\left\langle y_{0}, \varphi_{i}\right\rangle_{L^{2}\left(\Omega_{1}\right)} \varphi_{i}(b) \\
& =2 \sum_{i=1}^{\infty} t^{\alpha-1} E_{\alpha, \alpha}\left(\lambda_{i} t^{\alpha}\right) \sin \left(\frac{i \pi}{2}\right) \int_{0}^{1} \sin (2 \pi x) \sin (i \pi x) d x \\
& =0 .
\end{aligned}
$$

Hence, $K_{\alpha} y_{0}(x)=0$. Consequently, the state $y_{0}$ is not weakly observable in $\Omega_{1}$. On the other hand, one has

$$
\begin{aligned}
K_{\alpha} \chi_{\omega_{1}}^{*} \chi_{\omega_{1}} y_{0}(x) & =\sum_{i=1}^{\infty} t^{\alpha-1} E_{\alpha, \alpha}\left(\lambda_{i} t^{\alpha}\right)\left\langle\chi_{\omega_{1}}^{*} \chi_{\omega_{1}} y_{0}, \varphi_{i}\right\rangle_{L^{2}\left(\Omega_{1}\right)} \varphi_{i}(b) \\
& =\sum_{i=1}^{\infty} t^{\alpha-1} E_{\alpha, \alpha}\left(\lambda_{i} t^{\alpha}\right)\left\langle y_{0}, \varphi_{i}\right\rangle_{L^{2}\left(\omega_{1}\right)} \varphi_{i}(b) \\
& =2 t^{\alpha-1} E_{\alpha, \alpha}\left(-\pi^{2} t^{\alpha}\right) \int_{\frac{1}{6}}^{\frac{1}{3}} \sin (2 \pi x) \sin (\pi x) d x \\
& =\frac{(3 \sqrt{3}-1) t^{\alpha-1} E_{\alpha, \alpha}\left(-\pi^{2} t^{\alpha}\right)}{6 \pi} \\
& \neq 0,
\end{aligned}
$$

which means that the state $y_{0}$ is weakly observable in $\omega_{1}$. Moreover, for

$$
\beta_{1}(x)=\left|y_{\mid \omega_{1}}^{0}(x)\right|-1<y_{\mid \omega_{1}}^{0}(x)
$$

and

$$
\gamma_{1}(x)=\left|y_{\mid \omega_{1}}^{0}(x)\right|+1>y_{\mid \omega_{1}}^{0}(x), \quad \forall x \in \omega_{1},
$$

we have $\chi_{\omega_{1}} y_{0}(x) \in\left[\beta_{1}(\cdot), \gamma_{1}(\cdot)\right]$ and system (15) and (16) is exactly $\left[\beta_{1}(\cdot), \gamma_{1}(\cdot)\right]$-observable in $\omega_{1}$. The proof is complete.

Let $\mathcal{G}_{1}$ be the set defined by

$$
\mathcal{G}_{1}=\left\{g \in L^{2}\left(\Omega_{1}\right) \mid g=0 \text { in } L^{2}\left(\Omega_{1}\right) \backslash\left[\beta_{1}(\cdot), \gamma_{1}(\cdot)\right]\right\} .
$$

From Lemma 3, we see that

$$
\varphi_{0} \longmapsto\left\|\varphi_{0}\right\|_{\mathcal{G}_{1}}^{2}=\int_{0}^{T}\left\|C H_{\alpha}(T-t) \varphi_{0}\right\|^{2} d t
$$


defines a norm on $\mathcal{G}_{1}$. Consider the system

$$
\begin{cases}\mathcal{Q}{ }_{t} D_{T}^{\alpha} \Theta(x, t)=A^{*} \mathcal{Q} \Theta(x, t)+\delta(x-b) z(T-t) & \text { in } \Omega_{1} \times[0, T], \\ \Theta(\xi, t)=0 & \text { on } \partial \Omega_{1} \times[0, T], \\ \lim _{t \rightarrow T^{-}} \mathcal{Q}_{t} I_{T}^{1-\alpha} \Theta(x, t)=0 & \text { in } \Omega_{1} .\end{cases}
$$

It follows from Theorem 2 that the equation $\Lambda: \varphi_{0} \longmapsto \mathcal{P}(\Theta(0))$ has a unique solution in $\mathcal{G}_{1}$, which is also the initial state $y_{0}$ observed between $\beta_{1}(\cdot)$ and $\gamma_{1}(\cdot)$ in the subregion $\omega_{1}$.

\section{Conclusions}

In this paper we have investigated the notion of regional enlarged observability for a time fractional diffusion system with Riemann-Liouville fraction derivative of order $\alpha \in(0,1]$. We developed an approach that leads to the reconstruction of the initial state between two prescribed functions only in an internal subregion $\omega$ of the whole domain $\Omega$. We claim that the results here obtained can be useful to real problems of engineering.

As future work, we plan to study problems of regional boundary enlarged observability and regional gradient enlarged observability of fractional order distributed parameter systems, and provide illustrative numerical examples.

Author Contributions: H.Z., A.B. and D.F.M.T. contributed equally to this work.

Funding: This research was funded by Académie Hassan II des Sciences et Techniques, Morocco, grant number 630/2016 and by Fundação para a Ciência e a Tecnologia, Portugal, grant number UID/MAT/04106/2013 (CIDMA).

Acknowledgments: This research was initiated during a one-month visit of Zouiten to the Department of Mathematics of the University of Aveiro (DMat-UA), November and December 2017, followed by a ten days visit to DMat-UA in February 2018. The hospitality of the host institution and the support of Moulay Ismail University, Morocco, and R\&D unit CIDMA, Portugal, are here gratefully acknowledged. The authors would like also to thank two anonymous reviewers for their critical remarks and precious suggestions, which helped them to improve the quality and clarity of the manuscript.

Conflicts of Interest: The authors declare no conflict of interest.

\section{References}

1. Leibniz, G.W. Mathematische Schriften. Bd. V: Die mathematischen Abhandlungen; Herausgegeben von C. I. Gerhardt, Georg Olms Verlagsbuchhandlung: Hildesheim, Germany, 1962.

2. Samko, S.G.; Kilbas, A.A.; Marichev, O.I. Fractional Integrals and Derivatives; Gordon and Breach Science Publishers: Yverdon, Switzerland, 1993.

3. Machado, J.A.T.; Kiryakova, V. The chronicles of fractional calculus. Fract. Calc. Appl. Anal. 2017, 20, 307-336. [CrossRef]

4. Heymans, N.; Podlubny, I. Physical interpretation of initial conditions for fractional differential equations with Riemann-Liouville fractional derivatives. Rheol. Acta 2006, 45, 765-771. [CrossRef]

5. Povstenko, Y. Linear Fractional Diffusion-wave Equation for Scientists and Engineers; Springer: Cham, Switzerland, 2015. [CrossRef]

6. Tenreiro Machado, J.A.; Mainardi, F.; Kiryakova, V.; Atanacković, T. Fractional calculus: D'où venons-nous? Que sommes-nous? Où allons-nous? Fract. Calc. Appl. Anal. 2016, 19, 1074-1104. [CrossRef]

7. Dzieliński, A.; Sierociuk, D. Fractional order model of beam heating process and its experimental verification. In New Trends in Nanotechnology and Fractional Calculus Applications; Springer: New York, NY, USA, 2010; pp. 287-294. [CrossRef]

8. Sierociuk, D.; Dzieliński, A.; Sarwas, G.; Petras, I.; Podlubny, I.; Skovranek, T. Modelling heat transfer in heterogeneous media using fractional calculus. Philos. Trans. R. Soc. Lond. Ser. A Math. Phys. Eng. Sci. 2013, 371, 20120146. [CrossRef] [PubMed] 
9. Dzieliński, A.; Sarwas, G.; Sierociuk, D. Time domain validation of ultracapacitor fractional order model. In Proceedings of the 49th IEEE Conference on Decision and Control (CDC), Atlanta, GA, USA, 15-17 December 2010; pp. 3730-3735. [CrossRef]

10. Dzieliński, A.; Sierociuk, D. Ultracapacitor modelling and control using discrete fractional order state-space model. Acta Montan. Slovaca 2008, 13, 136-145.

11. Dzieliński, A.; Sierociuk, D.; Sarwas, G. Some applications of fractional order calculus. Bull. Pol. Acad. Sci. Tech. Sci. 2010, 58, 583-592. [CrossRef]

12. Hilfer, R. (Ed.) Applications of Fractional Calculus in Physics; World Scientific Publishing Co., Inc.: River Edge, NJ, USA, 2000. [CrossRef]

13. Magin, R.L. Fractional Calculus in Bioengineering; Begell House Inc.: Danbury, CT, USA, 2006.

14. Tavares, D.; Almeida, R.; Torres, D.F.M. Fractional Herglotz variational problems of variable order. Discrete Contin. Dyn. Syst. Ser. S 2018, 11, 143-154. [CrossRef]

15. Debbouche, A.; Torres, D.F.M. Sobolev type fractional dynamic equations and optimal multi-integral controls with fractional nonlocal conditions. Fract. Calc. Appl. Anal. 2015, 18, 95-121. [CrossRef]

16. Mozyrska, D.; Torres, D.F.M. Minimal modified energy control for fractional linear control systems with the Caputo derivative. Carpathian J. Math. 2010, 26, 210-221.

17. Xue, R. Observability for fractional diffusion equations by interior control. Fract. Calc. Appl. Anal. 2017, 20, 537-552. [CrossRef]

18. Axtell, M.; Bise, M.E. Fractional calculus application in control systems. In Proceedings of the IEEE Conference on Aerospace and Electronics, Dayton, OH, USA, 21-25 May 1990; Volume 2, pp. 563-566. [CrossRef]

19. Baleanu, D.; Golmankhaneh, A.K.; Golmankhaneh, A.K. On electromagnetic field in fractional space. Nonlinear Anal. Real World Appl. 2010, 11, 288-292. [CrossRef]

20. Kilbas, A.A.; Srivastava, H.M.; Trujillo, J.J. Theory and Applications of Fractional Differential Equations; North-Holland Mathematics Studies; Elsevier Science B.V.: Amsterdam, The Netherlands, 2006; Volume 204.

21. Liu, Z.; Han, J. Integral boundary value problems for fractional order integro-differential equations. Dyn. Syst. Appl. 2012, 21, 535-547.

22. Liu, Z.; Sun, J. Nonlinear boundary value problems of fractional functional integro-differential equations. Comput. Math. Appl. 2012, 64, 3228-3234. [CrossRef]

23. Oustaloup, A. From fractality to non integer derivation: A fundamental idea for a new process control strategy. In Analysis and Optimization of Systems; Bensoussan, A., Lions, J.L., Eds.; Springer: Berlin/Heidelberg, Germany, 1988; pp. 53-64. [CrossRef]

24. Podlubny, I. Fractional differential equations. In Mathematics in Science and Engineering; Academic Press, Inc.: San Diego, CA, USA, 1999.

25. Li, C.; Zeng, F. Numerical Methods for Fractional Calculus; Chapman \& Hall/CRC Numerical Analysis and Scientific Computing, CRC Press: Boca Raton, FL, USA, 2015.

26. Li, C.; Yi, Q.; Kurths, J. Fractional Convection. J. Comput. Nonlinear Dyn. 2017, 13, 011004. [CrossRef]

27. Li, C.; Chen, A. Numerical methods for fractional partial differential equations. Int. J. Comput. Math. 2018, 95, 1048-1099. [CrossRef]

28. Abbas, S.; Benchohra, M.; N'Guérékata, G.M. Topics in fractional differential equations. In Developments in Mathematics; Springer: New York, NY, USA, 2012; Volume 27. [CrossRef]

29. Agarwal, R.P.; Baleanu, D.; Nieto, J.J.; Torres, D.F.M.; Zhou, Y. A survey on fuzzy fractional differential and optimal control nonlocal evolution equations. J. Comput. Appl. Math. 2018, 339, 3-29. [CrossRef]

30. Zhang, X.; Zhong, Q. Triple positive solutions for nonlocal fractional differential equations with singularities both on time and space variables. Appl. Math. Lett. 2018, 80, 12-19. [CrossRef]

31. Amouroux, M.; El Jaï, A.; Zerrik, E. Regional observability of distributed systems. Int. J. Syst. Sci. 1994, 25, 301-313. [CrossRef]

32. Dolecki, S.; Russell, D.L. A general theory of observation and control. SIAM J. Control Optim. 1977, 15, 185-220. [CrossRef]

33. El Jaï, A.; Pritchard, A.J. Capteurs et actionneurs dans l'analyse des systèmes distribués. In Recherches en Mathématiques Appliquées [Research in Applied Mathematics]; Masson: Paris, France, 1986; Volume 3, p. 204.

34. El Jaï, A.; Simon, M.C.; Zerrik, E. Regional observability and sensor structures. Sens. Actuators A Phys. 1993, 39, 95-102. [CrossRef] 
35. Zerrik, E.H.; Bourray, H. Gradient observability for diffusion systems. Int. J. Appl. Math. Comput. Sci. 2003, 13, 139-150.

36. Ge, F.; Chen, Y.; Kou, C. Regional gradient controllability of sub-diffusion processes. J. Math. Anal. Appl. 2016, 440, 865-884. [CrossRef]

37. Debbouche, A.; Torres, D.F.M. Approximate controllability of fractional nonlocal delay semilinear systems in Hilbert spaces. Int. J. Control 2013, 86, 1577-1585. [CrossRef]

38. Debbouche, A.; Torres, D.F.M. Approximate controllability of fractional delay dynamic inclusions with nonlocal control conditions. Appl. Math. Comput. 2014, 243, 161-175. [CrossRef]

39. Karite, T.; Boutoulout, A.; Torres, D.F.M. Enlarged controllability of Riemann-Liouville fractional differential equations. J. Comput. Nonlinear Dyn. 2018, 13, 090907. [CrossRef]

40. Ge, F.; Chen, Y.; Kou, C. On the regional gradient observability of time fractional diffusion processes. Autom. J. IFAC 2016, 74, 1-9. [CrossRef]

41. Ge, F.; Chen, Y.; Kou, C. Regional Analysis of Time-Fractional Diffusion Processes; Springer: Cham, Switzerland, 2018. [CrossRef]

42. Zheng, G.; Ali, M.M. Observability estimate for the fractional order parabolic equations on measurable sets. Abstr. Appl. Anal. 2014, 361904. [CrossRef]

43. Lions, J.L. Contrôlabilité exacte, perturbations et stabilisation de systèmes distribués. Tome 1. In Recherches en Mathématiques Appliquées [Research in Applied Mathematics]; Masson: Paris, France, 1988.

44. Lions, J.L. Sur la contrôlabilité exacte élargie. In Partial Differential Equations and the Calculus of Variations; Birkhäuser Boston: Boston, MA, USA, 1989; Volume 2, pp. 703-727.

45. Almeida, R.; Pooseh, S.; Torres, D.F.M. Computational Methods in the Fractional Calculus of Variations; Imperial College Press: London, UK, 2015. [CrossRef]

46. Liu, Z.; Li, X. Approximate controllability of fractional evolution systems with Riemann-Liouville fractional derivatives. SIAM J. Control Optim. 2015, 53, 1920-1933. [CrossRef]

47. Mainardi, F.; Paradisi, P.; Gorenflo, R. Probability distributions generated by fractional diffusion equations. arXiv 2007, arXiv:0704.0320.

48. Klimek, M. On Solutions of Linear Fractional Differential Equations of a Variational Type; Czestochowa University of Technology: Czestochowa, Poland, 2009.

49. Pritchard, A.J.; Wirth, A. Unbounded control and observation systems and their duality. SIAM J. Control Optim. 1978, 16, 535-545. [CrossRef]

50. Salamon, D. Infinite-dimensional linear systems with unbounded control and observation: A functional analytic approach. Trans. Am. Math. Soc. 1987, 300, 383-431. [CrossRef]

51. Weiss, G. Admissible observation operators for linear semigroups. Israel J. Math. 1989, 65, 17-43. [CrossRef]

52. Curtain, R.F.; Zwart, H. An Introduction to Infinite-Dimensional Linear Systems Theory; Texts in Applied Mathematics; Springer: New York, NY, USA, 1995; Volume 21. [CrossRef]

53. Zouiten, H.; Boutoulout, A.; El Alaoui, F.Z. On the regional enlarged observability for linear parabolic systems. J. Math. Syst. Sci. 2017, 7, 79-87. [CrossRef]

54. Gorenflo, R.; Kilbas, A.A.; Mainardi, F.; Rogosin, S.V. Mittag-Leffler Functions, Related Topics and Applications; Springer Monographs in Mathematics; Springer: Heidelberg, Germany, 2014. [CrossRef]

(C) 2018 by the authors. Licensee MDPI, Basel, Switzerland. This article is an open access article distributed under the terms and conditions of the Creative Commons Attribution (CC BY) license (http:/ / creativecommons.org/licenses/by/4.0/). 